\title{
Strategies for Promoting Inclusivity in Health Sciences Education
}

\author{
Michele A. Haight ${ }^{1} \cdot$ Ingrid Bahner $^{2} \cdot$ Andrea N. Belovich $^{3} \cdot$ Giulia Bonaminio $^{4} \cdot$ Anthony Brenneman $^{5}$. \\ William S. Brooks ${ }^{6}$. Cassie Chinn ${ }^{7}$. Nehad El-Sawi ${ }^{8}$. Sandra B. Haudek ${ }^{9} \cdot$ Uzoma Ikonne $^{10} \cdot$ Robert J. McAuley $^{11}$. \\ Douglas McKell ${ }^{12} \cdot$ Rebecca Rowe $^{13} \cdot$ Mark Slivkoff $^{3} \cdot$ Tracey A. H. Taylor $^{11} \cdot$ Richard C. Vari $^{14}$
}

Accepted: 14 August 2021 / Published online: 10 September 2021

(C) International Association of Medical Science Educators 2021

The Spring 2021 Webcast Audio Seminar Series (WAS) of the International Association of Medical Science Educators (IAMSE) entitled, "Strategies for Promoting Inclusivity in Health Sciences Education," identified guiding principles for diminishing bias and promoting diversity, inclusivity, and equity in health sciences education. These guidelines included detailed analyses of classroom content and clinical practices and provided robust definitions for inclusivity, equity, and bias and commonly associated terms. Leading experts from a variety of health professions presented five weekly webinars to an international audience from March 4th through April 1, 2021.

\section{Creating Diverse, Equitable, and Inclusive Content in Medical Education}

\section{Presenter: Amy Caruso Brown, SUNY Upstate Medical University, Syracuse, NY, USA}

Dr. Amy Caruso Brown introduced this series by defining the concepts of diversity, equity, inclusion, and bias in health sciences education. She noted that these are complex notions informed by a compendium of different perspectives.

Michele A. Haight

mfhaight@gmail.com

1 Sam Houston State University College of Osteopathic Medicine, Conroe, TX, USA

2 Morsani College of Medicine, University of South Florida, Tampa, FL, USA

3 Idaho College of Osteopathic Medicine, Meridian, ID, USA

4 University of Kansas School of Medicine, Kansas City, KS, USA

5 University of Iowa Carver College of Medicine, Iowa City, IA, USA

6 University of Alabama at Birmingham School of Medicine, Birmingham, AL, USA
She defined bias as "an inclination or prejudice for or against something," and categorized the most common occurrences of bias in health sciences education into the following four areas: (1.) those admitted to the program and those hired to teach. (2.) The type and scope of content that is presented. (3.) The manner in which learners are mentored and evaluated, and (4.) faculty interaction with patients and families. Dr. Caruso Brown focused her comments and recommendations on educational content. She described the following four types of bias in curricular content: (1.) scientifically inaccurate information, (2.) over-emphasis on certain topics at the exclusion of others, (3.) promotion of bias, stigma, and shame towards people belonging to certain groups, and (4.) commentary that is irrelevant to medicine and promotes bias and discrimination. She also identified several biases that commonly occur in multiple choice exams. These include the following: (1.) use of race as a proxy for genetics, (2.) providing epidemiologic data without context, and (3.) use of male and female stereotypes. Dr. Caruso Brown noted that current curricular and assessment paradigms in health sciences education tend to obscure biases and make them difficult to identify. She provided the following link as a tool for identifying potential content biases: https://tinyurl. com/UpstateBiasChecklist. Dr. Caruso Brown suggested using

7 International Association of Medical Science Educators, Huntington, WV, USA

8 Des Moines University Medicine \& Health Sciences, Des Moines, IA, USA

9 Baylor College of Medicine, Houston, TX, USA

10 Eastern Virginia Medical School, Norfolk, VA, USA

11 Oakland University Beaumont School of Medicine, Auburn Hills, MI, USA

12 College of Our Lady of the Elms, Chicopee, MA, USA

13 University of New England College of Osteopathic Medicine, Biddeford, ME, USA

14 Virginia Tech Carilion School of Medicine, Roanoke, VA, USA 
the following methods to address content biases: (1.) remove content, (2.) replace content with images that challenge stereotypes, (3.) add content, and (4.) apologize. As a final recommendation, she suggested mapping the attributes of hypothetical patients used in the curriculum to help identify potential biases across the curriculum and to highlight opportunities to enhance equity and inclusion within the curriculum.

\section{Strategies to Recognize and Address Implicit or Explicit Bias in Small Group Teaching}

\section{Presenters: Karen Eley Sanders and Charlotte Baker, Virginia Tech Carilion School of Medicine, Roanoke VA, USA}

The second session of this series focused on "microaggressions" resulting from bias. Dr. Karen Eley Sanders began her presentation by showing a video with actors who commit "microaggressions" in a small group learning activity. Dr. Eley Sanders explained that microaggressions occur within a "system of bias." She described how this system is activated by a person making assumptions about stereotypes that can lead to prejudice; acting on prejudice can lead to discrimination, which can eventually lead to committing microaggressions. She shared Dr. D. Wing Sue's definition of microaggressions as "brief, everyday exchanges that send denigrating messages to certain individuals because of their group memberships." She underscored that microaggressions can be intentional or unintentional, but added that, regardless of intent, microaggressions have both short- and long-term effects on a person's mental health. Dr. Charlotte Baker followed up with a review of the same video, pausing to analyze each example of a microaggression. She provided alternative communication strategies to help avoid committing microaggressions. She cautioned that even if one person is not offended by certain comments, others might take offense. Drs. Eley Sanders and Baker concluded the session by providing the following recommendations for preventing microaggressions in health professions education: (1.) create a "safe space" in the learning environment to have open discussions about these types of issues, and (2.) acknowledge and apologize for any missteps in order to role model the types of behaviors desired from the learners.

\section{Achieving Equity in Assessment for Clinical Learners}

\section{Presenters: Arianne Teherani and Karen Hauer, University of California San Francisco School of Medicine, San Francisco, CA, USA}

During the third session of the series, Drs. Arianne Teherani and Karen Hauer explored the concept of equity within clinical assessment practices. Dr. Teherani began by identifying the foundational principles of equity that inform clinical education. These included the following: (1.) acknowledging that differences are not deficits, (2.) supporting the success of all learners, (3.) implementing multiple types of assessments, (4.) holding all learners to the same high standards, (5.) recognizing the moral, legal, economic, political, and social dimensions of assessment, and (6.) establishing a shared definition for an equitable outcome. She proposed a conceptual framework for equity in clinical assessment based on the following tenets: closing achievement gaps, equal treatment, fairness and inclusion, and justice. Within this framework, she described two levels of solutions, a macro level encompassing procedures/policies and mandates and a micro level encompassing one on one interactions. Dr. Teherani reviewed the evidence related to equity and clinical performance assessment and highlighted two areas of bias: differential achievement (divergence of educational outcomes for different demographic groups) and an amplification cascade (small differences in assessed performances lead to larger differences in grades).

Dr. Hauer proposed that addressing equity in clinical assessment requires an integrated, systems-based approach that focuses on the following: redesign of medical school curricula, redesign of learning and assessment methods, faculty development programs to identify and avoid bias, the use of frequent observation and feedback, the use of narrative assessments over time, and the avoidance of comparing learners. She identified three components of equity in assessment: intrinsic equity (the way one designs and conducts assessment practices), contextual equity (the environment in which learning and assessment occur), and instrumental equity (how assessment information is used to determine honors/awards). She juxtaposed older, "fixed, decontextualized" models of assessment practices with newer, "mastery models" of assessment practices that focus on a growth mindset through immersion learning. She noted that transitioning to newer assessment models would require faculty to think differently in order to overcome old assumptions. She underscored factors that influenced the performance of Underrepresented in Medicine (UiM) learners. These included stereotype threats and microaggressions. Dr. Hauer offered the following guidance for faculty who witness microaggressions: (1.) pre-brief learner responses to microaggressions, (2.) recognize and analyze microaggressions, (3.) consider different types of responses, (4.) respond in real time, (5.) check in with the learner, and (6.) follow-up with the learner. Dr. Hauer concluded the session with the following recommendations for achieving equity in assessment: (1.) examine your own data, (2.) design your assessment to fit your purpose, (3.) continuously improve assessment processes and practices, (4.) think of assessment as a system, and (5.) consider intrinsic, contextual, and instrumental aspects of equity in assessment. She provided the following resource to help 
guide efforts to establish equity in assessment: https://meded. ucsf.edu/faculty-educators/equity-assessment-guidelines-andchecklist.

\section{Pathways and Pipelines: Approaches to Increasing Diversity in the Health Professions}

\section{Presenters: Shani Fleming, University of Maryland Baltimore Graduate School, Baltimore, MD, USA, Norma Iris Poll-Hunter, Association of American Medical Colleges, Washington DC, USA, Rick McGee, Northwestern University School of Medicine, Chicago, IL, USA}

The fourth session of this series targeted promoting and sustaining inclusive practices across health sciences education. Three pre-eminent leaders from different health professions provided an overview of diversity and inclusion practices within their respective professions. Ms. Shani Fleming provided a brief history and overview of current Physician Assistant (PA) programs. She reported that the PA profession in the USA is predominantly white and female. She also noted that current trends show a troubling decrease in diversity and emphasized the need for collaboration with existing pipeline programs to increase opportunities for underrepresented students in the health professions. She highlighted the development of the Physician Assistants of Color (PAC) program, a grassroots movement dedicated to increasing diversity in the PA profession. She stated that effecting real change requires a systematic examination of health professions' culture through an anti-racist lens to identify and address disparities. She noted that this might include dismantling policies, practices, and procedures that are exclusionary for students of color. She concluded by recommending moving beyond "best practices," in order to create innovative pathways for underrepresented students.

Dr. Iris Poll-Hunter redirected the discussion to examine the medical education pipeline and the impact of these pipelines on the physician training continuum. She noted that, over the last 5 years, as the number of seats for medical students has increased, the number of students who are underrepresented in medicine (UiM) has remained stagnant. She further reported that over the past 30 years, $80 \%$ of entering medical students have come from families whose incomes are at the top socioeconomic quintiles. She proposed that educational equity involves examining the intersections of underrepresentation in race, ethnicity, and income. She noted that pipelines are important in attracting non-typical medical students, but cautioned that they cannot exist in isolation. Dr. Poll-Hunter's concluded her presentation by sharing the following lessons learned from premed pipelines: (1.) pre-med pipelines are essential because they increase diversity, which is a part of the solution, (2.) bundling activities is an important strategy that makes a difference, (3.) faculty engagement is critical because it predicts success, (4.) national and local partnerships are critical to sustainability, (5.) pipelines promote and increase diversity in the national pool and provide the ability to track participants, (6.) growing local talent from the community is essential to success, and (7.) all pipelines need to have wellness integrated into their programs.

Dr. Rick McGee provided an overview of diversity in research training and careers. He noted that $\mathrm{PhD}$ students are compensated to do their $\mathrm{PhD}$ training and are more like residents because they enter the workforce after their second year of training. He noted that $\mathrm{NIH}$ funding drives $\mathrm{PhD}$ training and provided a brief description of the genesis and current state of diversity efforts in $\mathrm{PhD}$ training. He highlighted the ongoing diversity programs such as the Society for the Advancement of Chicanos/Hispanic and Native Americans in Science (SACNAS), the annual Biomedical Research Conference for Minority Students (ABRCMS), and the AAMC Group on Graduate Research, Education and Training (GREAT). However, he reported that data suggest even with these programs, the number of NIH funded PIs has not changed for those who are supported by these programs. He also pointed out that underrepresented faculty numbers have not significantly increased over the past 40 years. He emphasized that simply getting underrepresented minority students into $\mathrm{PhD}$ programs is not a sufficient solution. He described more recent efforts to increase diversity, which include diversity supplements, mentored career development awards (K), enhanced emphasis on $\mathrm{PhD}$ training, enhanced mentor training with a focus on inclusion, a MOSAIC program designed to support the transition to faculty positions, and focused support through academic societies. He highlighted recently established diversity efforts such as the Fellowship in Research and Science Teaching (FIRST) which supports cluster hires, and NIH UNITE, which is designed to end structural racism and achieve racial equity. Dr. McGee stressed that efforts need to go beyond "numbers only" in terms of diversity and focus on equity and inclusion.

\section{Surviving Club Quarantine: Establishing Mentorship and Maintaining Wellness in a Diverse Student Population}

\section{Presenters: Marquita S. Norman, Wake Forest University School of Medicine, Wintston Salem, NC, USA, Kara L. Caruthers, University of Tennessee Health Science Center, Memphis, TN, USA}

The fifth and final session in this series underscored the role of wellness in the workplace for graduate health professions students. Dr. Marquita Norman categorized three facets of wellness: mentorship (talking with you), coaching (talking 
to you), and sponsorship (talking about you). She identified seven dimensions for wellness, but focused her commentary on the following four dimensions: emotional, intellectual, social, and occupational. She provided a list of key considerations for creating and sustaining an effective wellness program in the workplace. These included the following: (1.) explicit teaching or role modeling with respect to the culture and environment of higher education, (2.) recognition of the prevalence of Imposter Syndrome, especially among women and people of color, (3.) identifying policies for students' ability to work during training, (4.) identifying policies for addressing microaggressions, power dynamics, mistreatment, and the impact of COVID-19 on vulnerable populations, (5.) identifying and/or creating resources for psychological wellness, and (6.) providing faculty training in cultural humility and cultural sensitivity. In addition to these considerations, Dr. Norman emphasized that students with a sub-optimal K-12 or undergraduate education bring all of those experiences with them to the graduate health professions' workspace.

Ms. Kara Caruthers provided data on graduate health professions students' demographics. She noted that recent data indicate that there are more first-generation students, a wider age range of ethnic diversity, and increased variable income differences. She pointed out that any diversity, equity, and inclusion initiatives must address racism as a root cause. She suggested that this could be achieved through systematic review and revision of admissions policies, program curricula, clinical training, and remediation and retention policies. She emphasized that institutional change must occur through sustainable programs rather than performative gestures. She concluded by cautioning that a return to a post pandemic "normal" was not a reasonable solution because "normal" had not been working.

\section{Declarations}

Conflict of Interest The authors declare no competing interests.

Publisher's Note Springer Nature remains neutral with regard to jurisdictional claims in published maps and institutional affiliations. 